\title{
Scarff-Bloom-Richardson Grading System
}

National Cancer Institute

\section{Source}

National Cancer Institute. Scarff-Bloom-Richardson Grading System. NCI Thesaurus.

Code C113399.

A breast cancer grading system that was the precursor of the Nottingham system. 